\title{
阿司匹林水解反应动力学参数测定实验的改进
}

张胜红, 戚传松, 荣华, 龚良发, 李巍 ${ }^{*}$

北京石油化工学院化学工程学院, 北京 102617

摘要: 阿司匹林水解反应动力学是药学、医学类专业物理化学理论教学中的典型案例, 其动力学参数的测定通常采 用络合显色法, 存在实验步骤繁琐且耗时过长等问题, 难以转化为物理化学实验教学内容。为解决上述问题, 改进 了紫外分光光度法测定阿司匹林水解反应动力学的方法并详细探讨了其实验原理。和络合显色法相比, 新方案大幅 简化了实验内容和步骤, 避免了含酸废液的产生并将反应时间缩短为 4 小时。改进后的实验项目有望发展为药学和医 学类专业的化学动力学教学实验。

关键词: 阿司匹林; 乙酰水杨酸; 水解; 动力学; 分光光度法 中图分类号：G642.0；0643.1

\section{Improving Kinetic Measurements of Aspirin Hydrolysis}

\author{
Shenghong Zhang, Chuansong Qi, Hua Rong, Liangfa Gong, Wei Li * \\ School of Chemical Engineering, Beijing Institute of Petrochemical Technology, Beijing 102617, China.
}

\begin{abstract}
The kinetics of aspirin hydrolysis is the critical content of theoretical physical chemistry for both medical and pharmaceutical students. The experiment is usually conducted by monitoring aspirin's hydrolysis product (salicylic acid, SA) with a spectrophotometer after reaction with soluble $\mathrm{Fe}^{3+}$ salts. However, the kinetic measurement of aspirin hydrolysis has not been widely implemented in physical chemistry laboratory, because of the complicated procedures and the long testing period required to perform the reaction. In this work, the ultraviolet absorbance of SA at a wavelength of $296 \mathrm{~nm}$ has been employed to determine the kinetics of aspirin hydrolysis in a phosphate buffer $(\mathrm{pH}=$ 6.85). Compared with the traditional method, these improvements simplify the experimental procedures significantly, avoid the formation of liquid acidic wastes, and shorten the reaction time, thus, turn the hydrolysis of aspirin into a practical kinetic experiment for medical and pharmaceutical college students.
\end{abstract}

Key Words: Aspirin; Acetylsalicylic acid; Hydrolysis; Kinetics; Spectrophotometry

阿司匹林(又名乙酰水杨酸)是一种常见的非甾体药物, 广泛用于解热、阵痛、抗炎和抗血栓等方 面 ${ }^{[1]}$ 。阿司匹林的合成是有机化学的经典实验 ${ }^{[2-4]}$, 阿司匹林片剂的制剂、溶出和质量分析也是药 物化学及相关专业的综合性实验 ${ }^{[5]}$, 但其水解实验, 作为阿司匹林综合实验的重要一环, 至今尚未 纳入国内大学化学实验课程。究其原因, 现行阿司匹林水解反应动力学的测定主要基于络合显色 法 ${ }^{[6-9]}$, 即采用可溶性 $\mathrm{Fe}^{3+}$ 盐与阿司匹林水解产物水杨酸(见图1)在酸性条件下形成稳定的紫色络合 物, 然后利用可见分光光度法监测水杨酸络合物在波长 $525 \mathrm{~nm}$ 处的吸光度并间接测定阿司匹林的 含量。该方法在实验原理和操作层面存在以下不足:

收稿: 2020-10-23; 录用: 2020-11-19; 网络发表: 2020-12-30

“通讯作者, Email: liwei77@bipt.edu.cn

基金资助: 北京石油化工学院教育教学改革研究项目(ZD20160101) 
(1) $\mathrm{Fe}^{3+}$ 和水杨酸络合显色的 $\mathrm{pH}$ 值窗口较窄, 介于 2.5-3.5之间, 这直接导致可溶性 $\mathrm{Fe}^{3+}$ 盐存在时 只能测定酸性条件下阿司匹林水解反应的动力学数据, 难以模拟药物存储环境测定中性条件下阿司 匹林水解反应的动力学。

(2) 若采用反应液取样和 $\mathrm{Fe}^{3+}$ 络合显色, 实验操作过程繁琐, 需要准确移取一定量的反应液加入 到特定 $\mathrm{pH}$ 值(通常为缓冲溶液)和 $\mathrm{Fe}^{3+}$ 浓度的显色溶液; 此外, 该方法无法排除显色过程, 尤其是缓冲 溶液的低pH值(2.5-3.5)对阿司匹林水解反应的影响。

(3) 络合显色法需要事先明确络合物的稳定性、检出限和标准曲线的线性区间, 额外增加了实 验内容并延长了实验时间, 难以适应常规物理化学实验学时(4学时)的要求。

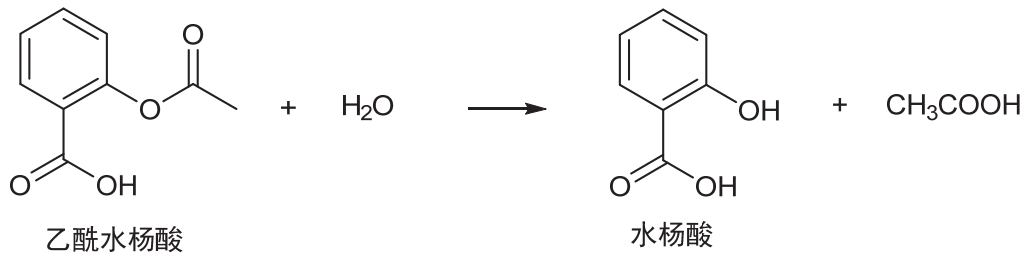

图1 阿司匹林水解反应方程式

为规避化学衍生显色法的弊端, 张彩云等 ${ }^{[10]}$ 基于水杨酸在 $275 \mathrm{~nm}$ 和 $316 \mathrm{~nm}$ 具有等吸收点的特 性, 采用双波长紫外分光光度法成功消除水杨酸对阿司匹林的干扰, 测定了阿司匹林水解反应的动 力学参数并据此推测阿司匹林水溶液的有效期。但该方法未采用缓冲溶液稳定反应体系的 $\mathrm{pH}$, 忽略 了产物水杨酸 $\left(\mathrm{p} K_{\mathrm{a}}=2.98\right)$ 对溶液 $\mathrm{pH}$ 以及阿司匹林水解速率的影响，在原理上存在瑕疵。

借鉴上述研究成果, 本文尝试从实验方法和内容方面进一步改进阿司匹林水解实验。具体而言, 采用近中性的 $\mathrm{NaH}_{2} \mathrm{PO}_{4}-\mathrm{Na}_{2} \mathrm{HPO}_{4}$ 缓冲体系 $\left(\mathrm{pH}=6.85,25^{\circ} \mathrm{C}\right)$ 模拟中性条件下阿司匹林水溶液的存储 环境, 采用紫外分光光度法监测产物水杨酸的吸光度并直接利用测得的吸光度替代浓度推导反应的 速率常数。改进的实验方案不仅简化了实验内容和操作过程, 缩短了反应时间, 而且在大幅度减少 药品使用种类和数量的同时避免了含酸废液的生成, 符合物理化学实验教学改革的要求。

\section{1 实验部分}

\section{1 实验原理}

阿司匹林和水杨酸的 $\mathrm{p} K_{\mathrm{a}}$ 分别为 3.57 和 2.98 , 在近中性的 $\mathrm{NaH}_{2} \mathrm{PO}_{4}-\mathrm{Na}_{2} \mathrm{HPO}_{4}$ 缓冲溶液中二者主要 以离子的形式存在并且对光的吸收集中在紫外区，如图2所示。

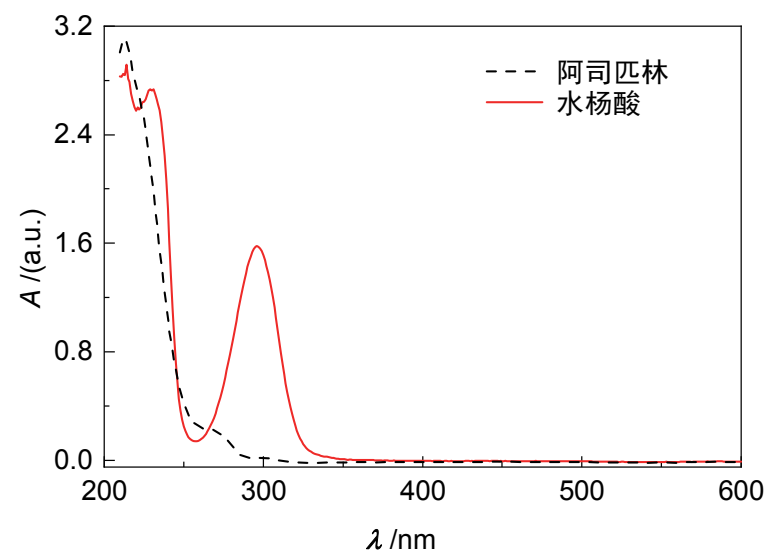

图2 阿司匹林和水杨酸在 $\mathrm{NaH}_{2} \mathrm{PO}_{4}-\mathrm{Na}_{2} \mathrm{HPO}_{4}$ 缓冲溶液中的紫外-可见吸收光谱 
其中, 水杨酸的最大吸收波长为 $296 \mathrm{~nm}$, 而阿司匹林在此波长时几乎不存在吸收(由于阿司匹林 在常温下亦能水解, 采用 $5 \times 10^{-4} \mathrm{~mol} \cdot \mathrm{L}^{-1}$ 的溶液实际测得水杨酸和阿司匹林在 $296 \mathrm{~nm}$ 处的摩尔吸光 系数之比大于 $100 / 1)$ 。因此, 理论上可以忽略阿司匹林的影响, 采用 $296 \mathrm{~nm}$ 波长时的吸光度 $A$ 监测水 杨酸的浓度以及阿司匹林水解反应的进度 ${ }^{[11]}$ 。

阿司匹林在 $\mathrm{pH}$ 恒定的水溶液中的水解反应为准一级反应, 反应速率与阿司匹林的浓度呈正比, 即:

$$
-\frac{\mathrm{d} c}{\mathrm{~d} t}=k c
$$

式中 $c$ 为时间 $t$ 时阿司匹林的浓度, $k$ 为速率常数。式(1)积分可得:

$$
\ln c=-k t+\ln c_{0}
$$

其中, $c_{0}$ 为阿司匹林的初始浓度。根据Lambert-Beer定律, 反应未开始、反应达到平衡和反应过 程中任意时间 $t$ 时溶液在 $296 \mathrm{~nm}$ 处的吸光度(记作 $A_{0}, A_{\infty}$ 和 $A_{t}$ ) 可以分别采用式(3-5)进行表示。考虑到 实验测定 $A_{\infty}$ 的数值需要等待较长的时间, 可以合理假设阿司匹林完全水解并采用浓度为 $c_{0}$ 的水杨酸 溶液的吸光度数值代替反应平衡时水解溶液的吸光度 $A_{\infty}{ }^{[11]}$ 。

$$
\begin{aligned}
& A_{0}=\varepsilon b c_{0} \\
& A_{\infty}=\varepsilon^{\prime} b c_{0} \\
& A_{t}=\varepsilon b c+\varepsilon^{\prime} b\left(c_{0}-c\right)
\end{aligned}
$$

式(3-5)中, $b$ 为石英样品池的内部宽度, $\varepsilon$ 和 $\varepsilon^{\prime}$ 分别为阿司匹林和水杨酸的摩尔吸光系数。将式 (3-5)联立, 得:

$$
\begin{gathered}
c_{0}=\frac{A_{\infty}-A_{0}}{b\left(\varepsilon^{\prime}-\varepsilon\right)} \\
c=\frac{A_{\infty}-A_{t}}{b\left(\varepsilon^{\prime}-\varepsilon\right)}
\end{gathered}
$$

进一步将式(6)和(7)代入式(2)得:

$$
\ln \left(A_{\infty}-A_{t}\right)=-k t+\ln \left(A_{\infty}-A_{0}\right)
$$

以 $\ln \left(A_{\infty}-A_{t}\right)$ 对 $t$ 作图, 由其斜率即可求得反应的速率常数 $k$ 。假设阿司匹林水解反应的活化能在 实验温度区间为定值, 则可由不同温度下的 $k$ 值和式(9)中的Arrhenius方程计算阿司匹林水解反应的 表观活化能 $E_{\mathrm{a}}$ 。

$$
\ln \frac{k_{2}}{k_{1}}=-\frac{E_{\mathrm{a}}}{R}\left(\frac{1}{T_{2}}-\frac{1}{T_{1}}\right)
$$

\section{2 实验仪器与试剂}

主要实验仪器: G-10双光束紫外可见分光光度计(让奇(上海)仪器科技有限公司); SYC-15c超级 恒温水浴(南京桑力电子设备厂); 电子分析天平，容量瓶， $100 \mathrm{~mL}$ 玻璃试管。

试剂: 阿司匹林, 水杨酸, $\mathrm{NaH}_{2} \mathrm{PO}_{4} \cdot 2 \mathrm{H}_{2} \mathrm{O}$ 和 $\mathrm{Na}_{2} \mathrm{HPO}_{4}$, 分析纯, 上海阿拉丁生化科技股份有限 公司; Parafilm封口膜, 瑞士Bemis公司。

\section{3 实验步骤}

(1) 配制溶液: 以 $\mathrm{NaH}_{2} \mathrm{PO}_{4}$ 和 $\mathrm{Na}_{2} \mathrm{HPO}_{4}$ 浓度均为 $0.025 \mathrm{~mol} \cdot \mathrm{L}^{-1}$ 的缓冲溶液为溶剂, 分别配制 $5 \times 10^{-4} \mathrm{~mol} \cdot \mathrm{L}^{-1}$ 的阿司匹林溶液和水杨酸溶液。

(2) 打开紫外-可见分光光度计, 待仪器稳定后以缓冲溶液为参照分别测定阿司匹林和水杨酸溶 液的紫外-可见吸收光谱, 确定水杨酸的最大吸收波长以及此波长时二者的摩尔吸光系数。

(3) 打开恒温水浴装置并设定目标温度为 $60{ }^{\circ} \mathrm{C}$, 待温度稳定后将盛有约 $60 \mathrm{~mL}$ 阿司匹林溶液的 大试管置入水浴中, 同时开始计时。每隔 $30 \mathrm{~min}$ 吸取适量反应液滴入石英比色血中并置于大量室温 水浴中冷却 $2 \mathrm{~min}$, 然后以缓冲溶液为参比测定反应液在 $296 \mathrm{~nm}$ 处的吸光度, 每个样品重复测量三次 
并对结果取平均值。反应时长控制为 $180 \mathrm{~min}$ 。

(4) 采用和步骤(3)相似的方法测定 $80^{\circ} \mathrm{C}$ 时阿司匹林水解反应液的吸光度, 取样间隔和反应时长 分别控制为 20 和 $80 \mathrm{~min}$ 。

\section{2 结果与讨论}

不同温度下阿司匹林水解反应液在 $296 \mathrm{~nm}$ 处的吸光度 $A$ 随时间 $t$ 的变化如图 3 (a)所示, 其中, $A_{0}$ 和 $A_{\infty}$ 分别为 0.0145 和 1.5991 。以 $\ln \left(A_{\infty}-A_{t}\right)$ 对 $t$ 作图, 结果如图3(b)所示, $60{ }^{\circ} \mathrm{C}$ 和 $80^{\circ} \mathrm{C}$ 的数据点均呈现明 显的线性相关(拟合直线的线性回归决定系数 $R^{2}$ 分别为 0.9999 和 0.9997 ), 证实上述条件下阿司匹林水 解遵从准一级反应动力学。由拟合直线斜率计算出 $60{ }^{\circ} \mathrm{C}$ 和 $80{ }^{\circ} \mathrm{C}$ 时阿司匹林水解反应的 $k$ 值分别为 $4.65 \times 10^{-3}$ 和 $2.03 \times 10^{-2} \mathrm{~min}^{-1}$, 进而根据式(9)中的Arrhenius公式得出阿司匹林在 $60-80{ }^{\circ} \mathrm{C}$ 近中性缓 冲溶液中水解反应的平均表观活化能 $E_{\mathrm{a}}$ 为 $72.0 \mathrm{~kJ} \cdot \mathrm{mol}^{-1}$ 。据文献报道 ${ }^{[12]}, \mathrm{pH}$ 为5-9时阿司匹林水解反 应的 $E_{\mathrm{a}}$ 为 $68.6-73.6 \mathrm{~kJ} \cdot \mathrm{mol}^{-1}$ 。改进实验得到的 $E_{\mathrm{a}}$ 值与文献结果吻合, 同时也接近络合显色法测得的弱 酸性介质中阿司匹林水解反应的活化能 $\left(69.8-73.0 \mathrm{~kJ} \cdot \mathrm{mol}^{-1}\right)^{[6-8]}$, 表明此改进实验方法的准确性能够 比拟传统络合显色法。
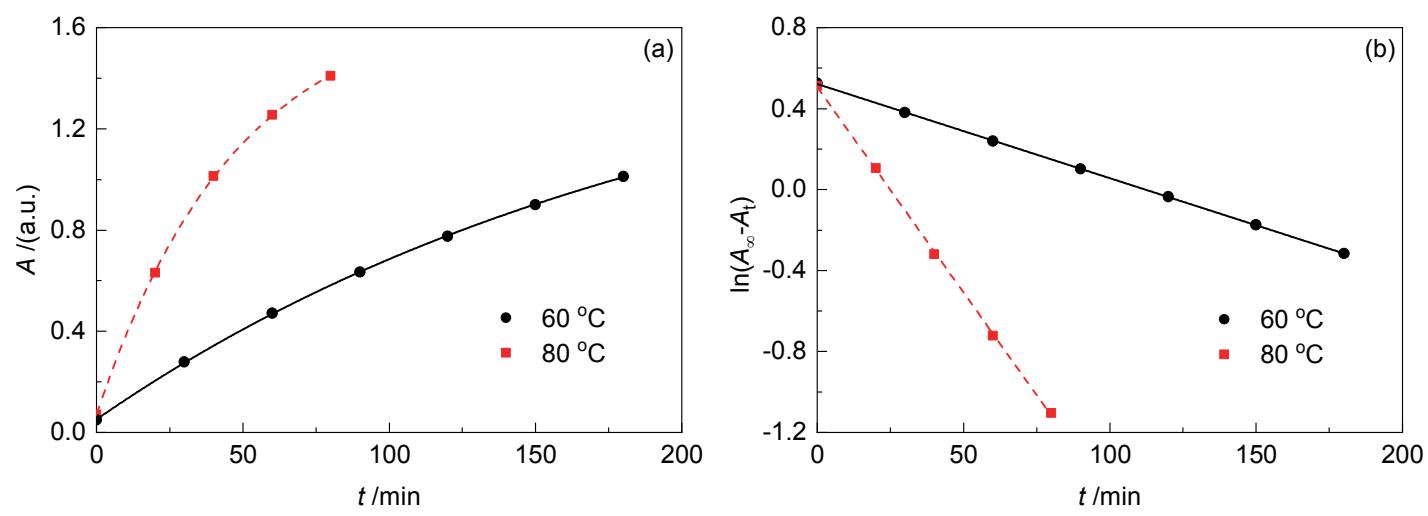

图3 不同温度下阿司匹林水解反应液吸光度 $A(a)$ 和 $\ln \left(A_{\infty}-A_{t}\right)(\mathrm{b})$ 随反应时间 $t$ 变化关系图

\section{3 实验教学建议}

\section{1 实验准备}

鉴于实验中阿司匹林和水杨酸溶液浓度较低 $\left(5 \times 10^{-4} \mathrm{~mol} \cdot \mathrm{L}^{-1}\right)$ 且用量不多 $(250 \mathrm{~mL}$ 和 $50 \mathrm{~mL})$, 直 接配制溶液容易导致较大的称量误差, 需要采用逐级稀释法配制。为减少学生配制溶液时的繁琐操 作和不必要的药品浪费, 建议任课教师或实验员提前准备足量的 $5 \times 10^{-4} \mathrm{~mol} \cdot \mathrm{L}^{-1}$ 的水杨酸溶液, 以 及 $\mathrm{NaH}_{2} \mathrm{PO}_{4}$ 和 $\mathrm{Na}_{2} \mathrm{HPO}_{4}$ 浓度均为 $0.025 \mathrm{~mol} \cdot \mathrm{L}^{-1}$ 的缓冲溶液。对于不稳定的阿司匹林溶液, 建议实验当 天配制浓度为 $5 \times 10^{-3} \mathrm{~mol} \cdot \mathrm{L}^{-1}$ 的母液以供学生稀释后直接使用。

\section{2 教学设计}

实验课堂教学中, 任课教师可以通过阿司匹林的 “一专多能” 功效激发学生的兴趣, 引导他们 思考: 为什么常见的阿司匹林药品是片剂而不是针剂或者口服液？进而引出化学稳定性是药物剂型 设计的关键因素, 以及如何设计实验测定阿司匹林水解反应的动力学参数。教学策略方面, 建议采 用探究式教学法鼓励学生质疑阿司匹林水解反应的准一级反应动力学特征并自己去证实, 以培养其 “质疑-假设-求证-结论” 的科学思维方法 ${ }^{[13]}$ 。具体而言, 任课教师不需要按部就班地讲解实验原 理、方法和内容, 而是应该鼓励学生对阿司匹林水解反应动力学进行大胆假设和小心求证。假设该 反应遵从一级反应动力学, 那么 $\ln c$ 应该与 $t$ 成线性关系, 也就是 $\ln \left(A_{\infty}-A_{t}\right)$ 与 $t$ 成线性关系; 然后指导 学生开展实验探究, 采集和分析数据以明确 $\ln \left(A_{\infty}-A_{t}\right)$ 与 $t$ 之间的关系。如果 $\ln \left(A_{\infty}-A_{t}\right)$ 与 $t$ 线性相关, 
即证实阿司匹林水解反应遵从一级反应动力学并由此计算相关的速率常数和表观活化能。

\section{3 实验拓展}

药品的有效期通常是指 $25^{\circ} \mathrm{C}$ 时药效损失 $10 \%$ 所需的时间。假设阿司匹林在 $60-80{ }^{\circ} \mathrm{C}$ 水解反应的 $E_{\mathrm{a}}$ 为常数, 则可进一步引导学生利用实验测定的数据估算阿司匹林溶液在 $25^{\circ} \mathrm{C}$ 和近中性条件下的有 效期。此外, 任课教师还可以要求学生思考并在实验报告中回答以下问题：(1) 阿司匹林溶液为什么 要现用现配? 其新鲜度对实验结果理论上有无影响? (2) 查阅文献, 列举至少三种测定阿司匹林水 解反应动力学的方法并评价其优缺点。(3) 查阅文献, 分析溶液 $\mathrm{pH}$ 值影响阿司匹林水解反应速率常 数的规律并给出初步解释。

\section{4 注意事项}

阿司匹林室温下在水中的溶解度较小, 配制溶液时可以采用超声辅助溶解; 为减少高温水浴时 阿司匹林溶液中水的蒸发, 非取样时间可以采用Parafilm封口膜对大试管进行密封; 为节约时间, 双 人实验小组成员在配制溶液前应预热分光光度计和恒温水浴, 组内分工分别测定 $60^{\circ} \mathrm{C}$ 和 $80^{\circ} \mathrm{C}$ 的反 应数据, 组间共用彼此的恒温水浴装置(即相邻两组的水浴温度分别设定为 $60^{\circ} \mathrm{C}$ 和 $80^{\circ} \mathrm{C}$ ) 以保证每组 同时进行两个温度系列的阿司匹林水解实验。

\section{4 结语}

借鉴现有阿司匹林水解反应动力学测定方法, 创新性地发展了单波长紫外分光光度法测定阿司 匹林水解反应动力学的实验方法。和传统络合显色法相比, 新方案大幅简化了实验内容和步骤, 双 人实验小组通过合理分工协作能够将实验时间控制在 4 小时以内。因此, 改进后的阿司匹林水解实验 有望发展成为面向药学和医学专业学生的化学动力学教学实验。

\section{参 考 文 献}

[1] 张芸溪, 高湖川, 张程瑞, 孙茂盛, 陈杨杰, 曾礼升, 黄岗, 陈鹏, 黄乾明. 有机化学, 2020, 40 (2), 300.

[2] 李立奇, 何友翔, 张元. 大学化学, 2018, $33(8), 43$.

[3] 康永锋, 马晨晨, 裴蓉. 实验技术与管理, 2016, 33 (10), 41.

[4] 段新红. 化学教育, 2016, 37 (24), 29.

[5] 夏金金, 孙继红, 李佳常, 李宁, 丁启龙. 药学教育, 2014, 30 (6), 78.

[6] 符小文, 张惠琼. 海南医学院学报, 1996, 2 (2), 58.

[7] 任晓棠, 王孜雁. 本溪冶金高等专科学校学报, 2004, 6 (2), 37.

[8] 张凯龙, 沈婷婷, 谭志文, 俞超. 实验室研究与探索, 2017, 36 (5), 32.

[9] 潘自红, 陈丽华, 梁媛媛, 单小芬. 分析试验室, 2013, 32 (9), 116.

[10] 张彩云, 董前年, 张传洋, 吴鸿飞, 倪佳, 李全, 鲁传华. 化学教育, 2015, 36(12), 32.

[11] Marrs, P. S. J. Chem. Educ. 2004, 81 (6), 870.

[12] Connors, K. A.; Amidon, G. L.; Stella, V. J. Chemical Stability of Pharmaceuticals, 1st ed.; John Wiley \& Sons, Inc.: Philadelphia, USA, 1986; pp. 222-223.

[13］李馨, 王海星, 张胜红. 化学教育(中英文), 2020, 41 (14), 37. 